and results from failure of fixation of the caecum and ascending colon, and is often associated with undue mobility of the right kidney (see Fig. 9).

But we must not ascribe all abnormal conditions of the stomach, duodenum, and gall bladder to ptosis. As already pointed out, many cases of duodenal obstruction are primarily due to the anatomical arrangement of the colic and mesenteric blood vessels, to periduodenitis, and other causes.

Some surgeons have taken extreme views. They have, however, done good service by directing attention to this condition, and a reasonable and judicial attitude must lead surgeons as a body to a correct line of treatment. This need not be heroic, nor must it lead to surgical neglect by ignoring conditions which are obviously present and demanding treatment. It must lead to surgical intervention on reasonable lines. In my view, those which should meet with gerieral acceptance are the division of bands when leading to local obstruction, shortcircuiting operations when the obstruction cannot be overcome in any other way, and the fixation of viscera on the right side the mobility of which is due to the failure of Nature to establish the usual anchorage. This is a surgical possibility, and the fixation of

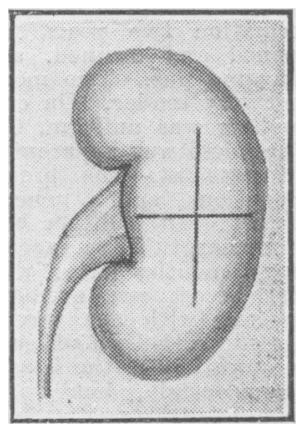

FIG. 10. may lead them either to advance odd doctrines or to oppose the introduction of any new ideas or methods of treatment. These extreme views are taken by different classes of surgeons. The first class I would call pioneers, though others, their strong opponents, might give them another name. Their opinions are of ten not accepted, but the expression of an extreme view startles people into thought, and it may be that in surgery, as in politics, the preaching of a startling doctrine is one of the surest ways to stimulate progress. But we have to bear in mind that what is newest is not necessarily best.

As opposed to the pioneers, we have the other extreme class whom we may call conservative

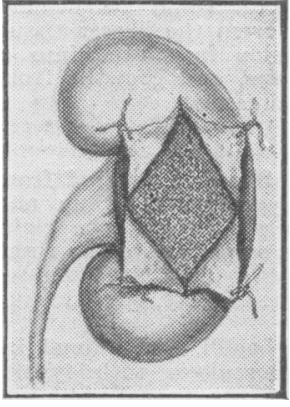
FIG. 11.
OPERATION OF NEPHROPEXY.

FIG. 10.-Incision in the capsule of the kidney.

FiG. 11.-Four flaps of kidney capsule reflected. surgeons, though their views are sometimes so negative that they give the impression that they have a habit of mind which refuses to accept anything new.

The general body of surgeons maintain a wise conservatism, a very different thing from "cld fogyism." As Osler forcibly pointed out, the motto of the one is, "Prove all things and hold fast that which is good," and of the other, "Prove nothing, but hold fast that which is old." But most surgeons are levelheaded and wait to see the result of clinical experience before committing themselves, realizing with Paget that that alone is true which the caecum, ascending colon, and right kidner will in many cases, though not in all, do much to relieve obstruction of the duodenum.

In those cases in which obstruction of the duodenum is caused by the root of the mesentery or the superior mesenteric vessels, fixation of the caecum and ascending colon will not give relief. Moreover, the weight of the small intestine cannot be taken off the root of the mesentery, and only a short-circuiting operation-either a gastrojejunostomy, or, probably better still, a duodenojejunostomy-will give reasonable prospect of cure.

In operating for right-sided ptosis $I$ make an incision, some eight to ten inches long, about an inch below and parallel with the line of the last rib. At the vertebral end this incision is curved upwards parallel with the erector spinae muscle, and a flap is reflected to expose the muscles covering the region of the last rib and last intercostal space. The peritoneum is opened in the front part of the incision, the condition of the caecum and ascending colon ascertained, and the appendix removed. The kidney is then delivered from the wound, and the renal pelvis and upper part of the ureter are freely exposed. The ureter and renal pelvis are carefully examined, any kinks are corrected, and adhesions or abnormal vessels causing kinks are divided. The ureter being now free and easily put in a straight position, the kidney is fixed. In my opinion, this should be done at a higher level than that usually practised, for pyelograms show that the upper calyces generally lie on the line of the last rib. I therefore make a crucial incision through the capsule of the kidney and reflect four flaps of capsule (Figs. 10 and 11). Sutures from the two lower flaps are tied round the last rib, holding the kidney up in a high position; sutures from the two upper flaps pass through the last intercostal space and hold the kidney steadily back (see Fig. 12). The caecum and ascending colon are then sutured to the posterior abdominal wall below the lower pole of the kidney, and in this way not only is fixation of the kidney rendered more secure, but the associated ptosis of the caecum and ascending colon is also corrected.

And now I will venture to deal with that other class of kinks to which $I$ referred at the beginning of this address. Lexicographers tell us that the word "kink," besides being used in the sense in which $I$ have used it in referring to the ureter and gastro-intestinal tract, is also applied to a crotchet, a whim, a caprice, or an odd and unreasonable notion. Surgeons are no more immune to this class of kink than other men and women. They may have acute or chronic mental kinks in one direction or another, which is proved clinically, and needs no other evidence. For medicine is the art of healing no less than the science of disease. Operative technique has now been so perfected, and such remarkable surgical feats can be performed with comparative impunity and trifling risk, that this very perfection has become a danger unless operative procedures are based on sound judgement. Where previously the risk would have deterred, now surgical judgement must control. We must be ever on the watch to prevent that judgement being biased, either by the odd and often ill founded recommendations of surgeons with kinks of exaggeration and enthusiasm, or by the attitude of the "old fogies" who would have us close our ears to anything new. For-

"Alas! what years you thus consume in vain,

Ruled by this wretched bias of the brain."-CRABBE.

\title{
TUBERCULOSIS OF THE BREAST.
}

$\mathrm{BY}$

NATHAN RAW, C.M.G., M.D., M.R.C.P.Lond.,

LORD CHANCELLOR'S VISITOR; HONORARY CONSULTING PHYSICIAN, PRESTON HALL COLONY FOR TUBERCULOUS EX-SERVICE MEN.

ONE of the most remarkable facts in the study of primary pulmonary tuberculosis is the extreme rarity of tuberculous mastitis. The breast tissue seems to be almost immune to attack by the human type of the bacillus. In progressive and advanced cases the lungs may be firmly adherent to the chest wall, with great involvement and thickening of the pleura, so that post mortem it is impossible to remove the lungs, yet the breast tissues are rarely involved. In the course of observations of tuberculosis during the last thirty years I have only met with seven cases of proved tuberculous mastitis-four in the female and three in the male. They were all very carefully investigated, and animal inoculations proved them to be tuberculous. In no case have I seen tuberculous mastitis associated with primary pulmonary tuberculosis.

According to Cornet, the infection rarely enters through the ducts of the glands; more often the bacilli enter by an excoriation, and are conveyed by the lymphatics, which accompany the galactic ducts. The infection, which is almost always unilateral, is followed by abscess formation with resulting sinuses.

In cows suffering from bovine tuberculosis the reverse is the case; tuberculosis of the udder is a very frequent 
complication, with resulting contamination of the milk by living bacilli.

The following is a very brief account of my seven cases:

\section{CASE I.}

A girl, aged 17, well nourished and robust, was admitted to hospital with extensive tuberculous glands on both sides of the neck. She had been operated on several times for removal and abscess formation, but the infection proceeded downwards to the axillary glands, and on the surface of the chest wall, towards the breast, formed which was opened and scraped. A persistent sinus resulted, which only healed after a long course of human tuberculin. The girl made a good recoverv, all the glands subsided, the sinuses and maders healed, and she was discharged from the hospital after ten months' treatment, apparently cured.

On examination of pus no tubercle bacilli could be demonstrated under the microscope, but scraping from the breast tissue showed them in large numbers. An emulsion inoculated into a the rabbit's spleen showed typical bovine cultures.

\section{CASE Ir.}

A woman, aged 37, seen in consultation. She had suffered from tuberculous neck glands for over twenty years, healing up and breaking down at intervals. A year ago she noticed the right axillary glands to be enlarged, the infection spreading to the right breast. The lungs were apparent of the breast, discharging were seen in the

Examination of the pus showed tubercle bacilli, and animal inoculation (rabbit) produced a general tuberculosis with typical bovine cultures. I advised amputation of the breast, but she declined, and I have not been able to learn what happened to her.

\section{CASE III.}

A girl, aged 19, was admitted to hospital with ver extensive tuberculosis of the right neck glands. She had been brought up in Canada and had drunk a very large quantity of cow's milk in Cana the last twelve years. The infection had spread by direct lymphatic extension to the axillary glands, which had suppurated, and thence to the breast, which was in a condition of general tuberculous mastitis with five sinuses discharging a large amount of pus daily.

The sinuses were scraped and packed with iodoform emulsion, and she was put on a course of human tuberculin. After a prolonged suppuration-ten months-they gradually closed, and she was sent to a sanatorium almost well. After a residence of six months, during which time she increased over 2 st. in weight, she was discharged with all sinuses and ulcers soundly healed, and she has remained quite free of the disease to the present time.

\section{CASE IV.}

A woman, aged 33, was admitted to hospital with extensive mastitis of the breast, numerous discharging sinuses, and a very fetid discharge.

History. At the age of 11 she first developed tuberculosis of the neck glands, which had been treated by incision and scraping on several occasions. There were several sinuses in the posterior triangle on the right side; the left side of the neck was quite clear. For over twenty years the glands had been more or less active and she had lost weight during the last six years. The infection had spread down the superficial lymphatics of the right infection had spread over the clavicle and direct to the breast. side of the chest, over the clavicle and direct to the breast
Numerous ulcers and scars were present over the chest wall.

Trcatment.-The sinuses were opened up and scraped and the cavities cleansed with a strong antiseptic, and afterwards packed with thymol. She was given a prolonged course of human tuberculin, and very soon began to show improvement. The progress was slow but progressive, and after a residence in hospital of fourteen months she was discharged, soundly healed, and has remained quite well up to the present time.

Case v.

A male, aged 27, was admitted to hospital suffering from tuberculosis of the shoulder (left). The head of the humerus had been excised seven years before, but the wound had never healed. He complained of pain, especially on pressure over the left breast. There was a uniform swelling of the breast tissue 3 inches iil diameter, with the nipple as the centre. There was fluctuation, and a needle showed thick pus; over an ounce was with-
drawn and found to be quite sterile. No tubercle bacilli could drawn and found to be quite
be seen under the microscope.

animal Inoculation.-A guinea-pig and a rabbit were inoculated with $1 / 2 \mathrm{c.cm}$. each of the pus, under the ckin of the abdomen. Both animals developed progressive tuberculosis and died in four months.

Post mortcm the rabbit showed a generalized tuberculosis of all the organs, with enormous caseation of the mesenteric glands. The guinea-pig showed a generalized infection, with deposits in liver and spleen, but no affection of lungs. Cultures made from the spleen of the rabbit on glycerin agar showed after six weeks a typical growth of bovine tubercle.

Progress of C'ase. - The abscess was evacuated with a trocar on three subsequent occasions, and disappeared. The patient left the hospital in two months, and I have not been able to trace him since.

Case vi.

A man, aged 23, has had tuberculosis of neck glands for seven years, and involvement of axillary glands for two years. The glands had been excised extensively, but repeated recurrences had involved further operations and scrapings. On admission he was thin and wasted, with all the appearances of lardaceous disease. His neck was one mass of ulcers and sinuses. The left axillary glands had suppurated and had been drained, leaving discharging sinuses. The left breast was enlarged, tender, but not uppurating.

Treatment.-The sinuses were treated antiseptically, but it was impossible to attempt anything of an operative character. The mastitis, however, gradually subsided, the pain on pressure disappeared, and the breast became normal in size. He gained weight, and left the hospital, but I have been unable to trace him since his discharge.

Case vir.

A male, aged 22, seen in consultation, complaining of pain in the abdomen, right knee, and right breast. He had suffered from tuberculosis of the knee-joint, tibia, and ankle, with abscess formation two years previously. The abscesses had all been opened and drained, and when seen all the sinuses were discharging pus. Two months before his right breast became large and very tender. On examination there was general mastitis, Swelling was uniform, there were

Treatment. -The breast was treated twice daily with iodex inunctions, and a general outdoor life with rest and tonic prescribed. In addition he was given a course of twelve injections of human tubercle vaccine in increasing doses. He was seen again three months later, when a great improvement was apparent. The breast was normal in size, there was no tenderness, the sinuses (with the exception of one over the ankle) had quite healed, and he had gained $22 \mathrm{lb}$. in weight. As there was no suppuration of the breast, it was not possible to prove its tuberculous origin, but $I$ have no doubt it was a case of early tuberculous mastitis, which was arrested. He is continuing to improve, and his doctor informs me that he is leading an ordinary life, and has returned to duty.

General Conclusions.

From a long clinical observation of tuberculosis in all ts forms, embracing over 10,000 cases in hospital and private practice, I have formed the following conclusions:

(1) Tuberculosis of the breast is always caused by the bovine bacillus.

(2) It is never primary, but is the result of lymphatic extension from the glands of the neck or axilla (excepting in the rare cases of general miliary infection).

(3) In common with all other forms of surgical tuberculosis caused by the bovine bacillus, it responds readily to treatment by a tubercle vaccine prepared from an attenuated culture of human tubercle bacilli. The treatment must, however, be continued for a long period until an active immunity is established.

\section{HAEMATEMESES "WITHOUT LESIONS."*}

BY

\section{GUSTAVE MONOD, M.D.PARIS, M.R.C.P.LoND., PHYSICIAN TO VICHY THERMAL HOSPITAL.}

I REPRESENT here the association of the Paris Faculty of Medicine for the furtherance of medical relations. It is our great desire to keep in touch with our British confreres. We will never forget the years we lived shoulder to shoulder.

International medicine has its organizations-the congresses-but times are hard, and we have been wondering if we might not find a simple bond of union by discussing simultaneously the same subject in the medical societies of the different countries. It is for this reason that $I$ am bringing before you a question which is being discussed at the present time by the Gastro-enterological Society of Paris, and is shortly to be brought before the Cercle Médical of Antwerp. The best proof $I$ can give of the opportunity of a working liaison between our countries is the fact that when I went through the literature of the subject I found that it had been very thoroughly investigated and discussed by our British colleagues.

I am seeking your criticisms and advice in answering the two questions: Is there such a thing as haematemeses without lesions? and, if so, how are such cases to be explained, and how should they be dealt with? In 1815 Latour published in Orleans a book-Histoire philosophique et médicale des hémorraghies - in which he recognized gastric bleeding sine materia, and quoted cases from Portal, Franck, Hoffmann, van Swieten, Baldinger, and Warton. The question had been raised at an even earlier date (1803) by Dalché. In

* A paper read to the Medical Section of the Royal Society of Medicina 